\title{
A novel steerable Foley balloon catheter for preventing phrenic nerve injury during epicardial catheter ablation
}

\author{
Naoki Yoshida • Yasuya Inden • Kyoko Soejima • \\ Hiromi Goto • Toyoaki Murohara
}

Received: 5 August 2013 / Accepted: 24 October 2013 / Published online: 16 February 2014

(C) Springer Science+Business Media New York 2014

Keywords Ventricular tachycardia $\cdot$ Epicardial catheter ablation $\cdot$ Subxiphoid pericardial window $\cdot$ Phrenic nerve injury

A 69-year-old man with a history of dilated cardiomyopathy underwent epicardial catheter ablation of repetitive ventricular tachycardia through a subxiphoid pericardial window. A perfect pace map was obtained at the lateral aspect of the left ventricular epicardium, but it was accompanied by phrenic nerve capture. We combined a 14-French Foley balloon catheter with a 5French steerable mapping catheter, (Fig. 1). The Foley balloon was then advanced into the pericardial space and navigated into position near the site of perfect pace map using the steerable mapping catheter. During the high-output pacing from the ablation catheter, the Foley balloon was inflated and resulted in the absence of phrenic nerve capture (Fig. 2). With the balloon inflated, irrigated radiofrequency current was delivered at that site. After the ablation, normal function of the left diaphragm was verified by fluoroscopy.

N. Yoshida $\cdot$ Y. Inden $(\bowtie) \cdot H$. Goto $\cdot$ T. Murohara Department of Cardiology, Nagoya University Graduate School of Medicine, 65 Tsurumai, Showa, Nagoya 466-8550, Japan

e-mail: inden@med.nagoya-u.ac.jp

K. Soejima

Department of Cardiology, Kyorin University School of Medicine, Tokyo, Japan

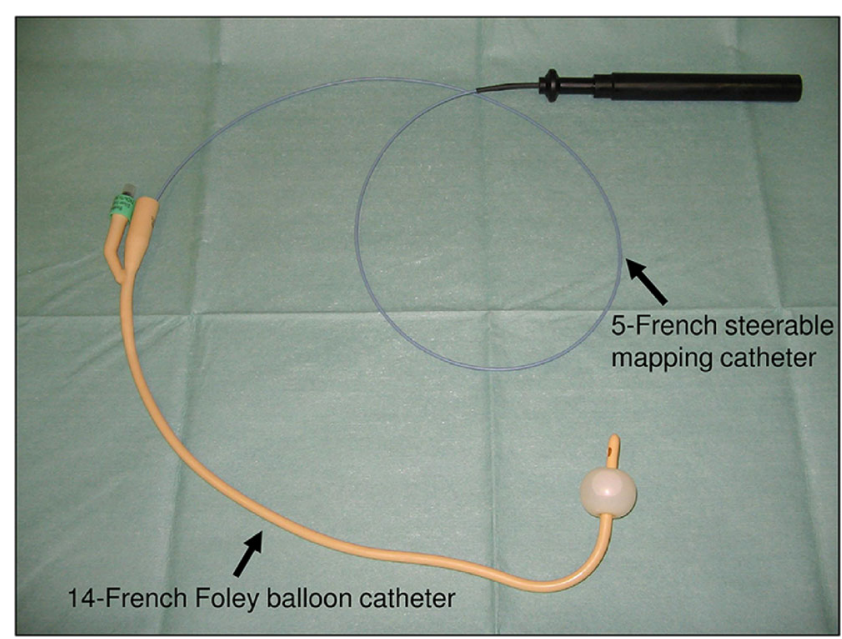

Fig. 1 A Foley balloon catheter (BARDEX ${ }^{\circledR}$ I.C., C. R. Bard, Murray Hill, NJ) combining with a steerable mapping catheter (WEBSTAR ${ }^{\circledR}$, Biosense Webster, Diamond Bar, CA)
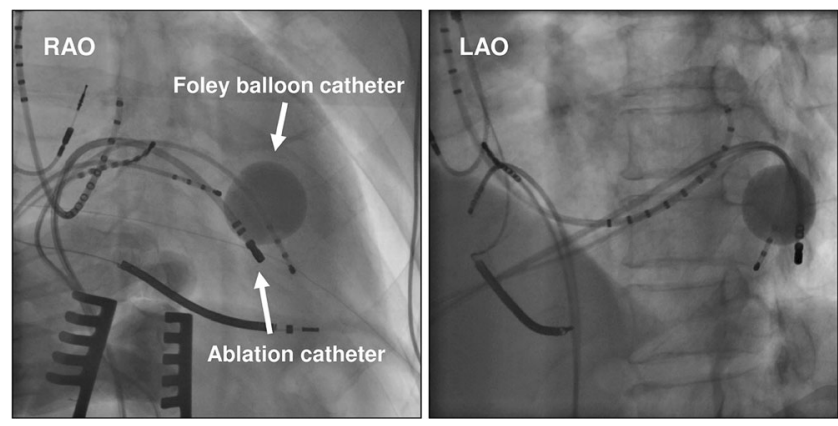

Fig. 2 Fluoroscopic images exhibiting the pacing site with phrenic nerve capture and the inflated Foley balloon catheter. $L A O$ left anterior oblique view; $R A O$ right anterior oblique view

Conflict of interest The authors declare that there are no conflicts of interest. No financial support was received for this study. 